\title{
Plan estratégico gerencial para empresas rentales en universidades públicas. Caso: REUNELLEZ
}

\author{
Chaparro, Luis* \\ Suárez, Madelein** \\ García, Tonny *** \\ Durán, Luis ${ }^{\star \star \star *}$
}

\begin{abstract}
Resumen
La empresa procesadora de granos REUNELLEZ-San Carlos, es una organización orientada a la obtención de recursos financieros destinados a suplir las deficiencias presupuestarias de la Universidad Ezequiel Zamora, mediante la prestación de servicios. Durante los últimos nueve años, la empresa ha presentado un flujo financiero deficiente, así como la falta de negocios alternativos que dinamicen sus actividades lucrativas. Es así como se diseñó una estructura metodológica, bajo los principios de Fred David (2005) y las perspectivas del cuadro de mando integral, para la mejora de la rentabilidad de la organización, soportado en un concepto estratégico y un diagnóstico interno, aplicando el instrumento FIM-Productividad (2004). Como resultado se obtuvo un $31 \%$ de cumplimiento de la normativa, indicando un desempeño deficiente en la evaluación interna. Respecto a los factores externos, se evidenció la posición frágil de la organización, con un total ponderado de 1,37 puntos. En la formulación de las estrategias, resultaron cuatro lineamientos tendentes a la diversificación concéntrica de las actividades y al mejoramiento de productos enfocados a la mejora de la rentabilidad.
\end{abstract}

Palabras clave: Plan estratégico, empresa rental, Reunellez, FIM-Productividad, Cuadro de mando Integral.

Recibido: 28-11-06. Aceptado: 22-10-07

* Profesor asistente. Procesos Agroindustriales I, Universidad Lisandro Alvarado, UCLA. Decanato de Agronomía. E-mail: luiscarloschaparro@gmail.com

** Profesor Asociado. Análisis y tratamiento de Aguas, Universidad Lisandro Alvarado. UCLA. Decanato de Agronomía. E-mail: jhannelypalmero@yahoo.es

*** Prof. Análisis de alimentos. UCLA. E-mail: tonnygarcia@ucla.edu.ve

**** Prof. Química de alimentos.UCLA. E-mail: winchox@yahoo.es 


\title{
Strategic Management Plan for Income-Producing Businesses at Public Universities. Case: REUNELLEZ
}

\begin{abstract}
The grain processing company, REUNELLEZ-San Carlos, is an organization devoted to obtaining funds destined to overcome budgetary deficiencies at the Universidad Ezequiel Zamora by rendering services. During the last nine years, the organization has had a deficient cash flow as well as a lack of alternative businesses that affect its lucrative activities. A methodological structure was designed, based on the principles of Fred David (2005) and the perspectives of an integral scorecard to improve the organization's profitability, supported by a strategic concept and an internal diagnostic, applying the FIM-Productivity instrument (2004). Results fulfilled $31 \%$ of the norm, indicating a deficient performance in internal evaluation. Regarding external factors, the organization's fragile position was shown with a weighted total of 1.37 points. In strategy formulation, the results were four lines tending toward the concentric diversification of activities and product improvement focused on improving profitability.
\end{abstract}

Key words: Strategic plan, income-producing business, Reunellez, FIM-Productivity, integral scorecard.

\section{Introducción}

Uno de los aspectos más discutidos en los últimos años a nivel nacional, en el ámbito de la Educación Superior, está referido a la autonomía de las universidades públicas; impulsada por las continuas crisis presupuestarias que las han aquejado desde siempre, y que exige una utilización del presupuesto más eficiente y una calidad de profesionales mucho mas aptos a la realidad del país. Terán (2002) señala: "El mayor desafío de la Educación Superior está referido a la necesidad de insertarse y orientar el cambio de la sociedad, en el aspecto económico y tecnológico". Para ello, la universidad debe implementar estructuras simples y flexibles en lo académico, científico y profesional así como mecanismos de financiamiento confiable y oportuno, que le permitan mejorar su funcionamiento, pero siempre preservando la autonomía de las instituciones.
En lo que respecta a la autonomía financiera de las instituciones públicas de Educación Superior, son muchas las propuestas que se han diseñado. Estas comprenden desde un cobro de matricula parcial, hasta el autofinanciamiento casi total de la institución, pero quizás una de las mas aceptadas y que se ha llevado a la práctica en algunas universidades a nivel nacional, consiste en el denominado "Conocimiento Aplicado" que no es más que la creación de estructuras para la generación de recursos financieros en base al dominio académico que se desarrolle en la institución, de tal forma de orientarlos al servicio de organizaciones públicas o privadas.

En el caso particular de la UNELLEZ- San Carlos, se logró constituir una empresa beneficiadora de granos (REUNELLEZ-San Carlos S.A.), destinada a la recepción, análisis, secado y almacenamiento de cereales como: Arroz, Sorgo y 
Maíz. REUNELLEZ inicia sus operaciones en el año 1987, teniendo como objetivo principal generar recursos financieros mediante la prestación de servicios agroindustriales en el ramo cerealero; así como de servir de ente difusor de la imagen institucional. Desde su fundación, y como normativa principal para su desempeño, la empresa REUNELLEZ - San Carlos S.A. debe realizar aportes financieros a la universidad, por el orden del 20 por ciento del total de sus utilidades netas generadas durante todo el año fiscal, con lo que la universidad intenta paliar sus deficiencias presupuestarias y necesidades inmediatas de liquidez.

En los últimos nueve años, la empresa REUNELLEZ no ha cumplido totalmente con el aporte financiero establecido, debido a que ha presentado un flujo financiero deficiente, producto de la falta de negocios alternativos que dinamicen sus actividades lucrativas y a la escasez de mecanismos confiables que le permitan hacer un seguimiento de sus programas y procesos de direccionamiento a largo plazo. Esta situación quedó evidenciada en los registros contables llevados por la empresa, donde los aportes hacia la universidad se encontraban en el orden del 10 por ciento de las utilidades generadas. Todo esto propició la necesidad de realizar una investigación orientada al diseño un plan estratégico gerencial para la empresa REUNELLEZ- San Carlos S.A. con el fin de incrementar la rentabilidad de la organización.

A través de la presente investigación, se logró determinar los aspectos más influyentes que afectan el desempeño de este tipo de organizaciones de servicios, pertenecientes a instituciones pú- blicas de educación superior basado, principalmente, en el análisis de aspectos como: Estructura organizacional, producción y/o servicios, comercialización, mercadeo y finanzas en el contexto regional y nacional para la determinación de los indicadores de desempeño del plan; involucrando a los actores de éste mediante la asignación de responsabilidades.

El análisis interno de la empresa se llevó a cabo con una población finita conformada por un total de 15 personas, constituida por los gerentes, directores y empleados de la misma. En lo que respecta al estudio de los usuarios del servicio que presta la empresa, estos fueron seleccionados de una población de 30 productores agropecuarios, ubicados en los municipios Rómulo Gallegos y Ricaurte del estado Cojedes; de los cuales se seleccionaron aquellos que aportaran el mayor porcentaje de ingresos para la empresa (87 por ciento), con lo que la población de usuarios resultó definida en 12 clientes.

En cuanto al diseño del concepto estratégico de la organización, así como en la formulación de las estrategias del plan, se empleó la metodología planteada por Fred David (2005). La formulación de los objetivos estratégicos, así como para el diseño del sistema de indicadores, se llevaron a cabo a través del método de Norton y Kaplan (2002) del Cuadro de Mando Integral.

\section{Diseño del concepto estratégico de la organización}

Como resultado de la revisión de los estatutos de la empresa y la posterior puesta en práctica del taller de redacción del concepto estratégico, se pudo definir 
Plan estratégico gerencial para empresas rentales en universidades públicas Chaparro, Luis; Suárez, Madelein; García, Tonny y Durán, Luis

la misión y visión para la empresa REUNELLEZ, San Carlos S.A. proyectada hasta el 2011, quedando definida de la siguiente forma:

\section{MISIÓN}

"Servir como ente generador de recursos financieros por excelencia para la universidad Ezequiel Zamora, a través de actividades productivas de alta rentabilidad, mediante el procesamiento de cereales, teniendo como premisa la calidad en los productos generados con alta tecnología, en función a los requerimientos de nuestros clientes en los Llanos centro Occidentales; brindando de esta forma, la satisfacción de nuestros empleados y proyección institucional a la UNELLEZ."

VISIÓN 2011

Consolidaremos nuestra posición en el mercado regional de cereales y extenderemos nuestras actividades a la generación de nuevos productos y servicios orientados a nuestros clientes. Ampliaremos los aportes generados para la universidad a un $25 \%$ de las utilidades netas generadas por la empresa y contaremos con un sistema autónomo de administración descentralizado; con lo cual seremos una organización orientada al mercado, promoviendo la aplicación de conocimientos en el área comercial, tecnológica y gerencial. Así mismo asumiremos un sistema automatizado de indicadores que no permita el seguimiento de proyectos de la empresa. Desarrollaremos un portafolio de clientes y proveedores que permitan la colocación de nuestros productos en toda la región, así como incentivaremos y capacitaremos nuestro personal, a través de planes de desarrollo conjunto con la universidad.

A diferencia de la metodología propuesta por David (2003), con respecto a los nueve componentes principales de una filosofía de gestión, fue necesario alinear el concepto estratégico de la empresa REUNELLEZ San Carlos S.A., con la filosofía existente en la UNELLEZ, universidad a la cual pertenece. Esto debido a que, a pesar de que la empresa es manejada de forma independiente como organismo autónomo, su capital es perteneciente por entero a la universidad, por lo cual debe existir una correspondencia entre la empresa y la institución de educación superior.

Como resultado de la alineación del concepto estratégico se establecieron cuatro elementos novedosos para este tipo de empresas, entre ellos se pueden mencionar: Tipo de clientes, Mercado a cubrir, Productos a ofrecer, y finalmente el auto concepto de la organización; todos ellos necesarios para el éxito de empresas de servicios.

\subsection{Diagnóstico de los factores internos de la organización}

Resultado de la auditoria interna, fueron identificadas cuatro fortalezas y doce debilidades las cuales se muestran en la Tabla 1. En ella se puede apreciar que el total ponderado para la organización es de 2.13, lo cual evidencia una posición débil en cuanto a su desempeño operativo interno.

Luego de la aplicación de la norma FIM productividad (2004), en su nivel básico de auditoria, en ella se pudo constatar que de las trece áreas propuestas por 
Tabla 1

Matriz de evaluación de factores internos

\begin{tabular}{lccc}
\hline Factores Internos Claves & Valor & Clasificación & Ponderación \\
\hline $\begin{array}{l}\text { Fortalezas } \\
\text { 1. Respaldo institucional de la universidad. }\end{array}$ & 0,04 & 4 & 0,16 \\
$\begin{array}{l}\text { 2. Personal preparado y de calidad. } \\
\text { 3. Vías de acceso. }\end{array}$ & 0,1 & 4 & 0,40 \\
4. Infraestructura apta para operación. & 0,04 & 3 & 0.12 \\
\hline $\begin{array}{l}\text { Debilidades } \\
\text { 1. Discontinua operatividad rentable. }\end{array}$ & 0,08 & 4 & 0,32 \\
$\begin{array}{l}\text { 2. Falta de promoción de la empresa. } \\
\text { 3. Manejo administrativo centralizado. }\end{array}$ & 0,1 & 1 & 0,1 \\
4. Escasos registros de producción e indicadores & 0,04 & 2 & 0,08 \\
de gestión. & 0,05 & 1 & 0,05 \\
5. Pocos planes de mejoramiento continuo. & & 2 & 0,14 \\
6. Falta de filosofía de gestión. & 0,06 & 1 & 0,06 \\
7. Falta de planes de inversión a largo plazo. & 0,08 & 1 & 0,08 \\
8. No existen Dpto. de mercadeo y ventas & 0,06 & 2 & 0,12 \\
9. No existen grupos gerenciales interfuncionales. & 0,07 & 2 & 0,14 \\
10. No existen indicadores de gestión por Dpto. & 0,04 & 2 & 0,08 \\
11. No existe planes en investigación y desarrollo. & 0,06 & 1 & 0,06 \\
12. No se cuenta con políticas para proveedores & 0,06 & 2 & 0,1 \\
ni clientes. & & & 0,12 \\
\hline
\end{tabular}

Fuente: Elaboración propia (2005).

el instrumento de evaluación, sólo cuatro fueron las pertinentes a someterse al estudio ( $31 \%$ de cumplimiento), por estar claramente identificadas y explícitamente asumidas por la directiva de la organización.

De las fortalezas de mayor ponderación se pueden apreciar las correspondientes a la alta preparación del personal de la empresa y la adecuada infraestructura operativa; esto coincide con lo planteado por Iribarren (2000), en su modelo de administración para una extensión universitaria, en el que señala que uno de los factores de menor aprovechamiento en empresas generadoras de recursos en universidades públicas, lo representa la aplicación práctica del conocimiento que en éstas se desarrolla (Ver Tabla 1).

En cuanto a las debilidades más resaltantes, estas consistieron en los escasos registros de producción e indicadores de gestión de la empresa, así como una débil estructura en cuanto a 
Plan estratégico gerencial para empresas rentales en universidades públicas Chaparro, Luis; Suárez, Madelein; García, Tonny y Durán, Luis

las actividades de mercadeo y ventas, manifestando una escasa consolidación de sus actividades y negocios alternativos. De la misma forma, Torres y Torrealba (2000) en su propuesta estratégica para una empresa rental, ha encontrado una fuerte correspondencia entre las actividades de mercadeo y oferta de nuevos productos, con la capacidad de financiamiento y generación de recursos en empresas rentales.

\subsection{Diagnóstico de los factores externos que afectan a la organización}

Como lo muestra la Tabla 2, el elemento externo que afecta en mayor grado el desarrollo de la organización, lo representa el carácter temporal de los rubros que sirven de materia prima a la empresa, concentrándose sus ciclos de cosecha en los meses de Febrero y Septiembre, lo cual afecta de manera significativa el continuo desarrollo de las operaciones de la organización y por ende la generación de recursos. El total ponderado de la evaluación externa, resultó de 1.37 puntos, lo cual indica que la empresa rental no está capitalizando las oportunidades, ni evitando las amenazas externas.

En esta matriz que se muestra a continuación, se puede observar que la participación de la empresa en las políticas económicas oficiales como cooperativas $y$ mercados populares, representa la oportunidad de mayor importancia relativa que se presenta según el escenario agroindustrial actual. Este mismo resultado lo obtuvieron Torres y Torrealba (2002) en el diagnóstico estratégico del mercado de los cereales en el estado Cojedes, en la que los autores destacan la potencialidad de este sector mediante la prestación de servicio en este ramo. Así mismo, lo que representa una buena oportunidad, también es reflejado como una posible amenaza en la matriz de factores externos, ya que tal como lo señala la Confederación Nacional de Asociaciones de Productores Agropecuarios (FEDEAGRO, 2004) existe poca claridad de las políticas de los organismos rectores de la materia, lo cual conducen a cierto grado de incertidumbre en el mercado de los cereales.

\subsection{Diagnóstico de las fuerzas competitivas imperantes en el sector agroindustrial de los cereales}

En cuanto a la posición de la empresa REUNELLEZ S.A. respecto a organizaciones de igual capacidad operativa en la región, se pudo evidenciar su reducida capacidad competitiva, sobre todo en lo que respecta a la capacidad de almacenamiento, operatividad de la empresa y oferta de nuevos productos y/o servicios. Tal como lo señala Corredor (2001) la confiabilidad y la variedad en la oferta, son factores determinantes en el éxito de empresas de servicios.

Las características mostradas por la empresa REUNELLEZ S.A. como resultado del análisis de la matriz competitiva, son equivalentes a los encontrados por Torrealba (2003) quien señala que el servicio de acondicionamiento de materias primas destinadas al procesamiento por parte de la agroindustria nacional, debe realizarse con el mayor aporte de valor agregado posible, de manera de traducir este servicio en nuevas opciones de negocios alternativos. 
Tabla 2

Matriz de evaluación de factores externos EFE

\begin{tabular}{lccc}
\hline Factores Externos Claves & Valor & Clasificación & Ponderación \\
\hline $\begin{array}{l}\text { Oportunidades } \\
\text { 1. Participación en políticas oficiales con cooperativas, } \\
\quad \text { mercados populares, etc. }\end{array}$ & 0,1 & 2 & 0,2 \\
2. Desarrollo de Nuevos. Productos y servicios. & 0,07 & 3 & 0,21 \\
$\begin{array}{l}\text { 3. Zona de creciente actividad agroindustrial (15\%). } \\
\text { 4. Apoyo financiero del gobierno. }\end{array}$ & 0,08 & 1 & 0,08 \\
$\begin{array}{l}\text { 5. Facilidades de créditos de siembra. } \\
\text { 6. Tendencia al cierre de importaciones de rubros }\end{array}$ & 0,06 & 1 & 0,06 \\
$\quad$ agrícolas (8\%). & 0,07 & 2 & 0,14 \\
Amenazas & 0,06 & 2 & 0,12 \\
$\begin{array}{l}\text { 1. Políticas oficiales cambiantes } \\
\text { 2. Control de precios. }\end{array}$ & 0,1 & 1 & 0,1 \\
3. Temporalidad de los rubros. & 0,08 & 1 & 0,08 \\
4. Costo de repuestos de maquinarias. & 0,1 & 1 & 0,1 \\
5. Nuevas empresas competidoras. & 0,06 & 1 & 0,06 \\
6. Poca fidelidad de clientes & 0,08 & 1 & 0,08 \\
7. Crecimiento urbano en zona industrial. & 0,07 & 1 & 0,07 \\
& 0,07 & 1 & 0,07 \\
\hline
\end{tabular}

Fuente: Elaboración propia (2005).

Por otra parte, Torres (2002) señala que las actividades de acondicionamiento de cereales en el estado Cojedes, ofrece una amplia gama de negocios alternativos por tener un margen de crecimiento estimado del $18 \%$ para los próximos 5 años, soportado en una política de restricción de importaciones por parte de los organismos gubernamentales.

Por otra parte, se pudo determinar que las empresas procesadoras de arroz, maíz y sorgo, poseen una capacidad instalada subutilizada en lo concerniente al acondicionamiento de estos cereales de origen regional, lo cual bien puede ser aprovechado para mejorar la calidad y can- tidad de estos en favor de la oferta, disponibilidad y comercialización nacional, además del beneficio socioeconómico derivado del valor agregado de inmediato impacto regional y nacional (ver Tabla 3).

El potencial operativo mostrado por la empresa procesadora de granos REUNELLEZ S.A, permite inferir que existe una gran oportunidad de relanzamiento de esta empresa rental, basado en una estrategia de descentralización gerencial hacia su área de influencia inmediata en la región centroccidental, lo cual constituye una opción altamente viable desde los puntos de vista logístico y operativo. 
Plan estratégico gerencial para empresas rentales en universidades públicas Chaparro, Luis; Suárez, Madelein; García, Tonny y Durán, Luis

Tabla 3

Matriz de competitiva de la empresa REUNELLEZ San Carlos S.A.

\begin{tabular}{lccccccc}
\hline & \multicolumn{3}{c}{ REUNELLEZ } & \multicolumn{2}{c}{ PROBALCA } & \multicolumn{2}{c}{ AGROINLLA } \\
\hline Factores de éxito & Valor & Clasif & Punto & Clasif & Punto & Clasif & Punto \\
\hline Personal preparado & 0,09 & 4 & 0,36 & 4 & 0,36 & 4 & 0,36 \\
Temporalidad de los rubros & 0,15 & 3 & 0,45 & 3 & 0,45 & 3 & 0,45 \\
Operatividad de la empresa & 0,10 & 1 & 0,10 & 2 & 0,20 & 1 & 0,10 \\
Capacidad de almacenamiento & 0,10 & 1 & 0,10 & 3 & 0,30 & 2 & 0,20 \\
Infraestructura de control de & & & & & & & \\
producción y control de calidad & 0,07 & 3 & 0,21 & 2 & 0,14 & 2 & 0,14 \\
Filosofía de gestión & 0,08 & 1 & 0,08 & 1 & 0,08 & 1 & 0,08 \\
Nuevos productos y servicios & 0,08 & 1 & 0,08 & 4 & 0,32 & 1 & 0,08 \\
Mercadeo y ventas & 0,10 & 1 & 0,10 & 2 & 0,20 & 1 & 0,10 \\
Calidad del servicio & 0,15 & 4 & 0,60 & 3 & 0,45 & 3 & 0,45 \\
Capacidad de respuesta & 0,08 & 2 & 0,16 & 2 & 0,16 & 2 & 0,16 \\
Total & 1,00 & & 2,24 & & 2,66 & & 2,12 \\
\hline
\end{tabular}

Fuente: Elaboración propia (2005).

\section{Formulación de las estrategias del plan}

Las estrategias de acción resultantes del análisis matricial obedecen a una serie única destinadas a la diversificación y mejoramiento de productos y/o servicios ofertados. Estas se señalan a continuación:

1. Desarrollar una línea de producción de alimentos concentrados, en búsqueda de diversificar sus productos y explotar mercados alternativos.

2. Insertarse en los programas de desarrollo agrícola del estado, a través del servicio a las cooperativas y mercados de alimentos populares y otros servicios.

3. Establecer alianzas estratégicas con otras empresas del ramo a través de la creación de departamentos de mercadeo y ventas.
4. Incursionar en el mercado de siembra y financiamientos de productos agrícolas.

La serie de opciones resultantes, concuerdan con en concepto planteado por Kaplan y Norton (2002), el cual señala que las estrategias destinadas a la diversificación concéntrica, consisten en el mejoramiento o adición de productos o servicios relacionados con las actividades claves de la organización. Esa así como la estrategia más notable, luego de ser evaluada por medio de una matriz comparativa, consistió en la destinada a la participación de la empresa en los programas de desarrollo agrícola del estado; considerando todos los factores internos y externos que pudieran afectar estas decisiones. 


\section{Formulación de los objetivos e indicadores del plan estratégico}

A partir de la línea estratégica más atrayente, definida en la etapa anterior (estrategia número 2), se derivaron los objetivos estratégicos basados en las cuatro perspectivas del cuadro de mando integral, definidas como se señala en el Diagrama 1.

Así mismo, sobre la base de los objetivos definidos se desplegaron los indicadores de gestión, con la cual se pudieron establecer los indicadores tanto de resultado como de proceso, los responsables en el control y seguimiento de cada uno de los indicadores, así como la frecuencia de medición de dichos objetivos.

A continuación, se presentan en forma detallada, los 28 indicadores obtenidos y la manera de calcularlos según cada perspectiva considerada.

\section{Perspectiva Financiera:}

1. Nuevas fuentes de ingresos. (\#)

2. \% de ingresos por negocios Nuevos.

$\%$ I.N.N. $=\frac{\text { Ingresos por negocios nuevos (Bs.) } \times 100}{\text { Ingresos Totales }}$

3. Rendimiento del capital sobre la inversión.

$$
\text { R.S.I. }=\frac{\text { Utilidad Neta (Bs.) }}{\text { Activos Totales (Bs.) }}
$$

4. \% Crecimiento de las Utilidades.

$$
\begin{aligned}
& \% \text { Crecimiento de Utilidades }= \\
& \frac{(\text { Utilidades del año (Bs. })-1) \times 100}{\text { Utilidades año anterior }}
\end{aligned}
$$

5. Aporte anual a la universidad.

Aporte anual $=$ Utilidad Neta año actual x 0.20 (Bs.)

\section{Perspectiva del Cliente:}

6. Estudio de Satisfacción de Clientes (\#)

7. Clientes Nuevos (\#)

8. \% Crecimiento cartera de clientes

$$
\% \text { C.C.C. }=\frac{(\# \text { Clientes actuales })-1) \times 100}{\# \text { Clientes del año anterior }}
$$

\section{Diagrama 1 \\ Cuadro de mando integral de la empresa REUNELLEZ San Carlos S.A.}

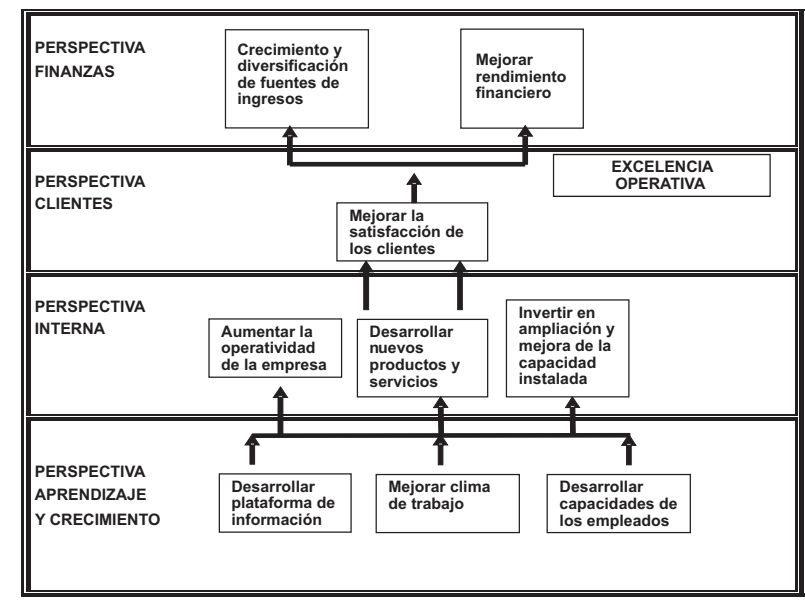

Fuente: Elaboración propia (2005). 
Plan estratégico gerencial para empresas rentales en universidades públicas Chaparro, Luis; Suárez, Madelein; García, Tonny y Durán, Luis

9. Eficiencia de Entrega

Eficiencia de Entrega =

(\# Pedidos entregados a tiempo) $\times 100$

$$
\text { Total de pedidos }
$$

10. Índice de Precio de Producto

I.P $=$ Precio promedio del mercado - Precio Producto

11. Quejas Recibidas (\#)

12. Sugerencias Implantadas (\#)

\section{Perspectiva Interna:}

13. Presupuesta Asignado a Inversiones (Bs.)

14. Inversión en mejoras y ampliación de capacidad instalada. (Bs.)

15. \% Cumplimiento de la inversión de capacidad instalada

$$
\% \text { P. M.C.I. }=\frac{\text { Bs. Invertidos en Mejoras x } 100}{\text { Bs. Asignados a mejoras }}
$$

16. Proyectos de Inversión Propuestos (\#) 17. Índice de continuidad.

$\mathrm{Ko}=\frac{\mathrm{Teq}}{\mathrm{Tt}}$

$$
\text { donde: }
$$

Teq = Tiempo efectivo de uso de maquinaria al año.

$\mathrm{Tt}=$ Fondo de tiempo total de uso de maquinaria/año.

18. Horas- hombre al año (\#).

19. Crecimiento Producción.

$$
\begin{aligned}
& \% \text { Crecimiento Producción }= \\
& \frac{\text { (Producción actual. Kg. ) }-1 \times 100}{\text { Producción año anterior }}
\end{aligned}
$$

20. Proyectos de Nuevos Productos y Servicios (\#)

21. Encuestas de Investigación de Mercado (\#)
22. Productos Nuevos Lanzados

$$
\text { al Mercado (\#) }
$$

23. \% De cumplimiento de la norma FIMProductividad.

\section{Perspectiva de Aprendizaje y crecimiento:}

24. Cursos de Capacitación (\#). 25. \% Empleados con cursos realizados.

$\%$ E.C.R. $=\frac{\text { (Número de empleados con cursos) } \times 100}{\text { Total de Empleados }}$

26.\% Empleados con bono de rendimiento.

$\%$ E. B.R. $=\frac{(\text { Empleados con bono de rendimiento }) \times 100}{\text { Total de empleados }}$

27. Control de avance de plataforma informática. (Tiempo programado)

28. Elaboración de base de datos. (Tiempo de elaboración de base de datos)

Con la aplicación del cuadro de mando integral, queda en evidencia la relación existente entre la estrategia central de la empresa y sus objetivos. Visto desde cuatro perspectivas, esto permitió definir cinco indicadores financieros, $y$ trece indicadores no financieros detallados de la siguiente forma: Siete indicadores referentes a la satisfacción de los clientes, once enfocados a los procesos operativos internos y cinco destinados al crecimiento y desarrollo del personal de la organización. Todos ellos, enfocados en el aumento de la rentabilidad de la empresa, pero desde áreas operativas diferentes. 


\section{Conclusiones}

La definición de la filosofía de gestión para organizaciones destinadas a la generación de recursos pertenecientes a universidades publicas deben estar alineadas con el concepto de la universidad a la cual pertenecen, por cuanto debe haber una clara correspondencia entre las actividades lucrativas de la empresa rental y los objetivos académicos, de promoción, extensión o de impacto social que se ese desarrolle en la institución educativa. De esta manera se instaura formalmente un grado de acompañamiento académico, que ampare las acciones operativas de la empresa, siempre y cuando no se pierda de vista el fin último que tiene una empresa rental, que no es otro que el de la generación de recursos financieros.

Organizaciones destinadas a la recepción, análisis y acondicionamiento de cereales, deben aplicar estrategias destinadas a la diversificación concéntrica de sus procesos, mediante la oferta de nuevos productos o servicios, esto motivado al carácter de disponibilidad temporal que presenta el ramo de los cereales, lo cual ejerce una influencia directa en la continua operatividad y con ello, fluctuaciones importantes en los ingresos percibidos.

La disminución de las importaciones en algunos rubros, conjuntamente con la iniciativa gubernamental de reactivar el consumo de bienes y servicios nacionales, constituye un factor determinante para la reactivación económica de la empresa REUNELLEZ, representando una fuente viable para la obtención de recursos con nuevas alternativas de negocios.
A pesar de su naturaleza de empresa rental, las organizaciones de este tipo, no deben definir sus objetivos de crecimiento únicamente enfocados al aspecto financiero de la organización, siendo el principal indicador la rentabilidad que se genera a través de sus actividades. La aplicación del Cuadro de Mando Integral, permiten generar un Plan Estratégico íntegro, que engloba cuatro elementos fundamentales para la empresa a saber: Aprendizaje y crecimiento, Clientes, Procesos internos, y Finanzas, cuyos indicadores derivados constituyen un esfuerzo conjunto, no solo en el área financiera sino en otras áreas de ejecución.

\section{Referencias Bibliográficas}

Corredor, Julio (2001). La Planificacion Estategica. Bases teóricas para su aplicación. Caracas, Venezuela. Editorial: Vadell Hermanos. Cuarta edición.

Confederación Nacional de Asociaciones de Productores Agropecuarios (FEDEAGRO). (2004). Página Web en línea. Disponible en: http://www.fedeagro.org.ve. Consulta [15/09/04].

David, Fred (2003). Conceptos de Administración Estratégica. Ciudad de México, Editorial Pearson Educación. Novena edición.

Kaplan, Robert y Norton, D. (2002). Cuadro de Mando Integral. Barcelona, España. Ediciones Gestión 2000. Segunda edición.

Iribarren, Daniel (2000). Modelo de Administracion para una Extension Universitaria. Caso: REUNELLEZ-San Carlos. Trabajo de ascenso. Universidad Nacional Experimental de los llanos Occidental Ezequiel Zamora. UNELLEZ San Carlos. 
Plan estratégico gerencial para empresas rentales en universidades públicas Chaparro, Luis; Suárez, Madelein; García, Tonny y Durán, Luis

Terán, Víctor (2002). Plan Estratégico para el Departamento de Ingeniería Industrial bajo la modalidad del Balanced Scorecard. Trabajo de Ascenso. Universidad Nacional Experimental Politécnica Antonio José de Sucre. UNEXPO-Barquisimeto.

Torrealba, Miguel (2003). Diseño de un plan estratégico para el desarrollo del sector caficultor en el estado Cojedes. Trabajo de grado de Maestría. Universidad Nacional Experimental Politécnica Antonio José de Sucre. UNEXPO-Barquisimeto.
Torres, Andrew y Torrealba, M. (2000). Propuesta estratégica para una empresa rental. UNELLEZ-San Carlos.

Torres, Andrew y Torrealba, M. (2002). Diagnóstico estratégico del Mercado Agroindustrial de Cereales del estado Cojedes. UNELLEZ-San Carlos.

Fundación para el mejoramiento de la productividad (FIM Productividad) (2004). Herramienta para el diagnóstico de la productividad en empresas venezolanas. 